\title{
Electron Beam Intraoperative Radiotherapy (ELIOT) in Pregnant Women with Breast Cancer: From in Vivo Dosimetry to Clinical Practice
}

\author{
Maria Leonardi ${ }^{a}$ Agnese Cecconi ${ }^{b, c}$ Rosa Luraschi ${ }^{d}$ Elena Rondid Federica Cattani ${ }^{d}$ \\ Roberta Lazzari $^{a}$ Anna Morra ${ }^{a}$ Santos Soto ${ }^{e}$ Vanna Zanagnolo ${ }^{f}$ Viviana Galimberti ${ }^{g}$ \\ Oreste Gentilini ${ }^{\text {g,h }}$ Fedro Peccatori ${ }^{i}$ Barbara Jereczek-Fossa ${ }^{a, c}$ Roberto Orecchia ${ }^{b, c, j}$
}

\footnotetext{
${ }^{a}$ Advanced Radiotherapy Center, European Institute of Oncology, Milan, Italy;

bScientific Direction, European Institute of Oncology, Milan, Italy;

c University of Milan, Milan, Italy;

dUnit of Medical Physics, European Institute of Oncology, Milan, Italy;

${ }^{e}$ Medica Sur Hospital Breast Center, Mexico City, Mexico;

${ }^{f}$ Gynecologic Cancer Surgery, European Institute of Oncology, Milan, Italy;

${ }^{g}$ Breast Cancer Surgery, European Institute of Oncology, Milan, Italy;

${ }^{\mathrm{h}}$ Current address: Breast Surgery Unit, San Raffaele University Hospital, Milan, Italy;

i Fertility and Procreation Unit, European Institute of Oncology, Milan, Italy;

j National Center of Oncology Hadrontherapy (CNAO foundation), Pavia, Italy
}

\section{Keywords}

Breast cancer · Pregnancy · Radiation therapy

\section{Summary}

Background: The aim of this study was to confirm our preliminary results with in vivo dosimetry in non-pregnant breast cancer patients receiving electron beam intraoperative radiotherapy (ELIOT) and to report on the first treatment in a pregnant woman. Patients and Methods: Following our previous experience, 5 non-pregnant patients receiving ELIOT to the tumor bed after breastconserving surgery (BCS) were studied with thermoluminescent dosimeters positioned in the subdiaphragmatic region, within the uterus, and in the ovarian region. In December 2011, the first pregnant breast cancer patient underwent BCS and ELIOT (21 Gy at $90 \%$ isodose) during the 15th week of gestation. Results: The mean dose to the subdiaphragmatic external region in the 5 nonpregnant patients was $5.57 \mathrm{mGy}$, while pelvic measurements were below $1 \mathrm{mGy}$. The actual dosimetry of the pregnant patient showed a mean subdiaphragmatic dose of $4.34 \mathrm{mGy}$, a mean suprapubic dose of $1.64 \mathrm{mGy}$, and mean ovarian doses of $1.48 \mathrm{mGy}$ (right-sided) and $1.44 \mathrm{mGy}$ (left-sided). The expected dose to the fetus was estimated as $0.84 \mathrm{mGy}(0.004 \%$ of the prescribed dose). Conclusion: ELIOT as an anticipated boost to the breast could be considered in pregnant women in the early second trimester, postponing whole-breast irradiation after delivery.

(C) 2017 S. Karger GmbH, Freiburg

\section{Introduction}

It is estimated that breast cancer occurs in 1 in 3,000 pregnancies and accounts for one-third of maternal deaths during gestation $[1,2]$. As the trend for delaying pregnancy into the later reproductive years continues, this rare association is likely to become more common. The therapeutic management of pregnant women with breast cancer is difficult and should be undertaken by a dedicated multidisciplinary team with sufficient expertise to meet the needs and requests of such a distressing clinical situation [3]. There are few reports in the literature addressing the issues related to radiotherapy during pregnancy [4-6]. Irradiation of the embryo/fetus during pregnancy can, in principle, cause the death of the embryo and increase the risk of deleterious effects in the newborn child [7, 8]. In the adjuvant setting after breast-conserving surgery (BCS), benefits and risks of irradiating the breast must be carefully consid-

\section{KARGER}

(c) 2017 S. Karger GmbH, Freiburg
Dr. Agnese Cecconi 
Table 1. Results for each in vivo dosimetry in non-pregnant patients $^{\mathrm{a}}$

\begin{tabular}{lllllllll}
\hline Patient & $\begin{array}{l}\text { Energy, } \\
\text { MeV }\end{array}$ & $\begin{array}{l}\text { Prescribed } \\
\text { dose, Gy }\end{array}$ & $\begin{array}{l}\text { Irradiated } \\
\text { breast }\end{array}$ & $\begin{array}{l}\text { Right ovary, } \\
\text { mGy }\end{array}$ & $\begin{array}{l}\text { Left ovary, } \\
\text { mGy }\end{array}$ & $\begin{array}{l}\text { Suprapubic, } \\
\text { mGy }\end{array}$ & $\begin{array}{l}\text { Subdiaphrag- } \\
\text { matic, mGy }\end{array}$ & $\begin{array}{l}\text { Uterus, } \\
\text { mGy }\end{array}$ \\
\hline 1 & 10 & 21 & left & 0.925 & 1.001 & 0.78 & 56.231 & 0.570 \\
2 & 8 & 21 & left & 0.453 & 0.443 & 0.38 & 1.639 & 0.300 \\
3 & 8 & 12 & left & 0.677 & 0.557 & 0.435 & 2.902 & 0.366 \\
4 & 8 & 21 & right & 0.881 & 0.814 & 0.682 & 1.980 & 0.485 \\
5 & 9 & 12 & left & 0.314 & 0.293 & 0.576 & 7.758 & 0.261 \\
\hline
\end{tabular}

aPeripheral doses (mGy) measured with shielded thermoluminescent dosimeters at subdiaphragmatic and pubic surfaces of patients in relation to radiation energy and irradiation instrument (Liac mobile linear accelerator). ered. Although the modern attitude is to offer the same surgical approach to pregnant women as to non-pregnant women [9], some concerns still remain about the delay in administering postoperative radiotherapy after childbirth. After BCS, intraoperative radiotherapy with electrons (ELIOT) $[10,11]$ might improve local control and allow a safe irradiation of the breast after delivery. In our previously published experience assessing in vivo dosimetry in the uterine cavity of non-pregnant women, doses to the abdomen were extremely low [12]. In the current study, we concluded the dosimetry phase, including 5 additional patients, and proceeded to the clinical phase, delivering ELIOT to a 15-week pregnant woman.

\section{Methods and Materials}

\section{In Vivo Study in Non-Pregnant Breast Cancer Patients}

Between February and April 2011, we performed in vivo dosimetry in 5 non-pregnant patients who underwent breast ELIOT, using micro-rod thermoluminescent dosimeters (TLDs) (TLD-100 ${ }^{\mathrm{TM}}$, Harshaw, Solon, OH, USA). ELIOT to the breast tumor bed was administered with a mobile linear accelerator (either Novac7, Hitesys, Aprilia, Italy or Liac, Info \& Tech, Rome, Italy) installed in the operating theater. Procedures and results of our previous in vivo dosimetry study were reported in 2008 [12]. In these 5 additional non-pregnant patients, in vivo dose measurements were extended to the ovarian region.

\section{ELIOT in a Pregnant Breast Cancer Patient}

In December 2011, the first pregnant patient was treated with ELIOT as a part of breast-conserving therapy. During regular breast cancer screening mammography, a 41-year old woman, gravida 1 para 1-0-0-1, was diagnosed with a suspicious small lesion in the left breast. The histologic result of an ultrasound-guided needle biopsy was grade 2 infiltrating ductal carcinoma. While being investigated for breast cancer, the patient found out that she was pregnant at $4-5$ weeks.

A multidisciplinary team dedicated to the management of fertility and pregnancy issues in oncology thoroughly discussed the treatment options with the patient, taking into account her preference to proceed with gestation and to have BCS.

After signing informed consent, quadrantectomy and sentinel node biopsy were performed with the aid of intralesional injection of ${ }^{99 \mathrm{~m}} \mathrm{Tc}$-labeled nanocolloid (sentinel node and occult lesion localization, i.e. SNOLL procedure). The final histologic report showed multifocal grade 2 ductal carcinoma with grade 2 ductal intraepithelial neoplasia (DIN 2) present at 1 margin, staged pT1 (maximum tumor size $1.5 \mathrm{~cm}$ ), pN0 (sentinel node), estrogen receptor $95 \%$ progesterone receptor $20 \%$, Ki-67 18\%, HER2/neu 0.

Because of the positive margin, a reoperation was performed 2 months later at 15 weeks gestation during which ELIOT was administered. No residual disease was found at re-excision. Technical parameters of ELIOT included $6 \mathrm{~cm}$ flat collimator, $6 \mathrm{MeV}$ electron energy, dose prescription of $21 \mathrm{~Gy}$ at $90 \%$ isodose.
TLDs were positioned on the patient abdomen as for non-pregnant women: subdiaphragmatically at $13.5 \mathrm{~cm}$ from the applicator, in suprapubic position at $24.5 \mathrm{~cm}$ from the applicator, and above the ovarian region at $26 \mathrm{~cm}$ (left-sided) and $28.5 \mathrm{~cm}$ (right-sided) from the applicator. As a substitute for intrauterine TLD, the 5 th dosimeter was positioned above the fetus, identified by ultrasound, at $27 \mathrm{~cm}$ from the applicator. A shielding apron (2-mm lead equivalent) was placed on the patient's abdomen to block most of the electron scatter from the machine.

\section{Results}

\section{In Vivo Study in Non-Pregnant Patients}

The in vivo dosimetry results in the non-pregnant patients are shown in table 1 . The beam energies used for irradiation were in the range $8-10 \mathrm{MeV}$, applicator diameters were $4-6 \mathrm{~cm}$. TLDs were placed on the skin as follows: subdiaphragmatically at a median distance from the ELIOT applicator of $20.4 \mathrm{~cm}$ (range 12-26 $\mathrm{cm}$ ), in the suprapubic position at a median distance of 43.7 (range $36-47 \mathrm{~cm}$ ) from the applicator, and at a median distance of $40 \mathrm{~cm}$ (range 29-47 cm) from the applicator for ovaries (left and right) identified by ultrasound. Finally, the intrauterine catheter of $30-\mathrm{cm}$ length was placed. A shielding apron (2-mm lead equivalent) was placed on the patients' abdomen to block most of the electron scatter from the machine. Taking as reference the dose of $21 \mathrm{~Gy}$, the mean dose to the subdiaphragmatic external region was of 5.57 $\mathrm{mGy}$, the mean dose to the suprapubic external region was 0.72 $\mathrm{mGy}$, the mean dose to the ovarian sites, right and left respectively, was 0.80 and $0.75 \mathrm{mGy}$, and the mean intrauterine dose was 0.49 $\mathrm{mGy}$. The results confirm our previously published experience assessing in vivo dosimetry [12] in which we measured mean doses of $3.7 \mathrm{mGy}$ (range 1-8.5 mGy) in the subdiaphragmatic region, 0.9 $\mathrm{mGy}$ (range $0.3-2 \mathrm{mGy}$ ) in the pubic region, and $1.7 \mathrm{mGy}$ (range 0.6-3.2 mGy) in utero. Note that for Patient 1 the reading for the subdiaphragmatic position is very high compared to other results; it was discovered that the TLD was not covered by the shielding apron.

\section{Data of the Pregnant Patient}

The TLD measurement results of the pregnant patient are shown in table 2.

The mean dose to the subdiaphragmatic external region at 13.5 $\mathrm{cm}$ from the applicator was $4.34 \mathrm{mGy}$; the mean dose to the suprapubic external region at $24.5 \mathrm{~cm}$ from the applicator was 1.64 
Table 2. Dosimetry results for the pregnant patient

\begin{tabular}{lllllll}
\hline $\begin{array}{l}\text { Prescribed } \\
\text { dose, Gy }\end{array}$ & $\begin{array}{l}\text { Irradiated } \\
\text { breast }\end{array}$ & $\begin{array}{l}\text { Energy, } \\
\mathrm{MeV}\end{array}$ & $\begin{array}{l}\text { Right ovary, } \\
\mathrm{mGy}\end{array}$ & $\begin{array}{l}\text { Left ovary, } \\
\mathrm{mGy}\end{array}$ & $\begin{array}{l}\text { Suprapubic, } \\
\mathrm{mGy}\end{array}$ & $\begin{array}{l}\text { Subdiaphrag- } \\
\text { matic, mGy }\end{array}$ \\
\hline 21 & left & 6 & 1.479 & 1.438 & 1.635 & 4.344 \\
\hline
\end{tabular}

$\mathrm{mGy}$, and the mean dose to the abdominal site near the right (distance from applicator $28.5 \mathrm{~cm}$ ) and left (distance from applicator $26 \mathrm{~cm}$ ) ovary was 1.48 and $1.44 \mathrm{mGy}$, respectively.

For each position, the patient received about twice the average dose received by the other 5 patients included in this study. Based on measurements calculated by excess, the uterus/fetus should have received $0.004 \%$ of the prescribed dose (21 Gy), i.e. $0.84 \mathrm{mGy}$. At the 39th week of gestation, the patient gave birth to a live healthy male infant appropriate for the gestational age, $4 \mathrm{~kg}$ in weight, with an Apgar score of 10/10. Soon after delivery, the patient started adjuvant endocrine therapy (triptorelin $3.75 \mathrm{mg}$ every 28 days intramuscularly and tamoxifen $20 \mathrm{mg}$ daily). She was advised not to breastfeed her child. 2 months after delivery, the patient received hypofractionated whole-breast radiotherapy using an intensity-modulated radiotherapy (IMRT) technique employing tomotherapy. The breast area irradiated with ELIOT was excluded from the external beam irradiation volume. The scheme comprised 8 fractions of $4 \mathrm{~Gy}$ each, up to a total dose of $32 \mathrm{~Gy}$, equivalent to a total dose of 42.7 Gy with the 2 Gy per fraction scheme $(\alpha / \beta=4)$. At the last follow-up after 5 years, the patient was free of disease and was satisfied with the cosmetic outcome. So far, her child has been in good health and has shown normal development based on age and size percentile.

\section{Discussion}

Full-dose ELIOT resulted in an estimated fetal dose of $0.84 \mathrm{mGy}$ ( $0.004 \%$ of the prescribed ELIOT dose). Although the well-accepted general rule is to avoid radiotherapy during pregnancy unless the tumor is threatening the mother's life [13], this value can be considered relatively low and therefore acceptable, considering the risk estimation reported in the scientific literature regarding the biological effects of radiation. However, ELIOT compares favorably regarding the well-known variables influencing the dose absorbed by a certain organ or structure at a distance [14]. In fact, as the peripheral dose increases with larger field size, greater depth of prescription, and higher energy used, ELIOT takes advantage of a small collimator diameter, low electron energy (maximum 10 $\mathrm{MeV}$ ), and short depth of dose penetration (maximum $2.5 \mathrm{~cm}$ ). For most electron beams, the Bremsstrahlung component is low both inside and outside the radiation field. In the dosimetric study evaluating external electrons in pregnancy performed by Antolak et al. [15], the scattered dose from the electron beam was supposed to be less than $1 \%$ of the prescription dose outside the field. According to the recommendations issued by radiation protection agencies [8], the risk is negligible if the fetal dose does not exceed 1 mGy, while doses of a few mGy are still considered acceptable. The additional doses received by the pregnant patient with mammography and the SNOLL procedure might be considered negligible also. In fact, the absorbed dose to the fetus in patients receiving lowdose lymphoscintigraphy with ${ }^{99 \mathrm{~m}} \mathrm{Tc}$ human serum albumin nanocolloids is estimated to be less than $10 \mu \mathrm{Gy}$ (0.01 mGy) [16], while in standard mammography the dose to the uterus is less than 0.03 $\mu \mathrm{Gy}(0.00003 \mathrm{mGy})$ per imaged breast during the first trimester [17]. The cumulative dose from diagnostic and therapeutic procedures for the fetus in this report remains within a few mGy. The strength of the ELIOT procedure is that it compares favorably with external breast adjuvant irradiation, where the estimated dose to the fetus ranged between 0.14 and 0.18 Gy (140-180 mGy) [18]. Regarding the effect of fractionation, the fetal absorbed single dose of ELIOT is lower than that of the daily dose per fraction given in long-course radiotherapy ( 0.84 vs. $5-8 \mathrm{mGy}$ per fraction considering a total dose of $50 \mathrm{~Gy}$ (25 fractions) [19]. Although the embryo/ fetus must be deemed sensitive to radiation at all stages of gestation, most biologic effects of radiation have a dose-response relationship. Even in the absence of a true dose threshold, some radiation-induced effects may be undetectable, falling within the range of the natural frequency of abnormalities. For deterministic effects, experimental data showed a sigmoid dose-response curve, with a threshold value of approximately $0.1 \mathrm{~Gy}$ (100 mGy) [20]. Although recommendations provided by an internal expert panel in 2010 [21] considered it safe to administer radiotherapy in the first trimester (provided that the distance between radiation field and embryo is adequate), we deemed it more prudent to avoid any therapeutic exposure until after the 10th week of gestation [22] when organogenesis is completed, following the general rule that postoperative breast radiotherapy is not an emergency treatment. Conversely, at the beginning of the second trimester, the fetus was at such a distance that low levels of scattered radiation were expected to be absorbed. As the peripheral dose decreases exponentially with increasing distance, the distance of the fetus from the field edge is one of the most important factors. With external beam radiotherapy using a 4-MV linear accelerator, Glasgow et al. [23] calculated doses of 500, 200, and $100 \mathrm{mGy}$ at 20,30, and $40 \mathrm{~cm}$ from the field edge, while Var der Giessen et al. [5] calculated doses of 200, 70, and $30 \mathrm{mGy}$ at the same distances. Although dosimetric measurement in non-pregnant women in the subdiaphragmatic region showed a low peripheral dose as well, in the third trimester of gestation it is considered more reasonable to postpone any form of radiotherapy, including ELIOT [24]. As far as stochastic effects are concerned, we cannot exclude the possibility that the lifetime risk for radiation-induced fatal cancer is higher than that expected without any intrauterine exposure. However, due to the low dose administered via ELIOT, it will probably be close to the normal incidence and indiscernible from the natural background [25]. 
Table 3. Probability of bearing healthy children as a function of radiation dose

\begin{tabular}{|c|c|c|}
\hline $\begin{array}{l}\text { Dose to embryo/ } \\
\text { fetus above the natural } \\
\text { background, mGy }\end{array}$ & $\begin{array}{l}\text { Probability of } \\
\text { no } \\
\text { malformation }\end{array}$ & $\begin{array}{l}\text { Probability of } \\
\text { no cancer } \\
(0-19 \text { years })\end{array}$ \\
\hline 0 & 97 & 99.7 \\
\hline 1 & 97 & 99.7 \\
\hline 5 & 97 & 99.7 \\
\hline 10 & 97 & 99.6 \\
\hline 50 & 97 & 99.4 \\
\hline 100 & 97 & 99.1 \\
\hline$>100$ & $<97$ & $\leq 99$ \\
\hline
\end{tabular}

In table 3 is reported the probability of bearing healthy children as a function of radiation dose recommended by the International Commission on Radiological Protection (ICRP 84) [25].

Although the existence of a dose threshold for carcinogenic effects of radiation has been debated, it is generally accepted that doses of the order of $10 \mathrm{mGy}$ increase the relative risk to 1.4 , although the absolute excess risk remains low [26]. Osei et al. [27], using a dedicated computer program for fetal dose and risk estimation, calculated that the absorbed dose from the majority of radiologic examinations lay in the range of $<0.001-21.9 \mathrm{mGy}$. With these values, the associated probability of both induced hereditary effects and childhood cancer $\left(<5 \times 10^{-9}\right.$ to $1 \times 10^{-4}$ and $<8 \times 10^{-8}$ to $2 \times 10^{-3}$, respectively) is lower than the natural baseline risk in human populations [28]. As the estimated absorbed dose to the fetus from ELIOT is comparable with that from extraabdominal radiologic examinations, no increase in the incidence of both hereditary disease and childhood cancer is expected. In non-pregnant patients, the risk of genetic abnormalities is commonly expressed as the gonadal dose. In our study population, the dose measured on the skin above the ovaries was in the range of $0.75-1.48 \mathrm{mGy}$. Since the risk of genetic effects is $0.5 \%$ per 1 Gy absorbed (1,000 mGy) [29], also with external beam breast radiotherapy where gonadal doses are reported to lie within the range of 37-63 mGy $[4,5,30]$, the risk of hereditary effects on the newborn's progeny is negligible compared with the natural incidence. Due to the young age and the tumor biology, the single dose of 21 Gy given intraoperatively (partial breast irradiation) to our patient was not considered fully adequate for local control $[31,32]$. Therefore, the patient underwent whole-breast irradia- tion with IMRT about 16 weeks after the delivery. Due to the foreseen time gap between ELIOT and whole-breast external beam radiotherapy, the intraoperative boost dose was increased to $21 \mathrm{~Gy}$, which is the conventional full dose used in partial breast irradiation. The IMRT technique was chosen to paint deliberate inhomogeneity across the breast, thus allowing a lower dose in the breast region already treated with full-dose ELIOT. A lower intraoperative boost dose, e.g. 12 Gy [10], allows delivering conventional $3 \mathrm{D}$ whole-breast radiotherapy with no need for complex techniques such as IMRT. Obviously, the optimal dose combination of the anticipated boost administered via ELIOT and further post-delivery whole-breast external beam radiotherapy in breast cancer diagnosed during pregnancy needs to be defined. Due to the long overall treatment time (boost and whole-breast treatment), the total dose and/or the dose of each component should probably be higher than that in conventional adjuvant breast irradiation in order to overcome interval tumor cell repopulation.

This dosimetric study suggests that ELIOT may be a reasonable option for pregnant women undergoing BCS during the late first and early second trimester. The main limitation lies in the lack of data regarding the efficacy of such an approach. The delay between the administration of this form of an anticipated boost and the commencement of whole-breast external beam radiotherapy might jeopardize local control [33]. On the other hand, the possibility for pregnant breast cancer patients to receive chemotherapy during their pregnancy, with the exception of the first trimester, might play a protective role in local control, allowing these patients to go through gestation with an active treatment and to have radiotherapy after delivery. Anthracyclines, cyclophosphamide, and taxanes have shown good clinical activity without considerable morbidity to the fetus $[34,35]$. The ELIOT anticipated boost is not meant to be a practice-changing approach but rather an option to be offered to pregnant women. This dosimetric study showed that it is feasible and relatively safe for the fetus, and that it could increase local control of the tumor bed which is considered the area most at risk of local relapse, until the patient can receive a more extensive radiotherapy treatment.

\section{Disclosure Statement}

All authors declare no conflict of interest.

\section{References}

1 Pentheroudakis G, Pavlidis N: Cancer and pregnancy: poena magna, not anymore. Eur J Cancer 2006;42: $126 \mathrm{e} 40$

2 Pavlidis NA: Coexistence of pregnancy and malignancy. Oncologist 2002;7:279-287.

3 Amant F, Han SN, Mhallem Gziri M, et al.: Management of cancer in pregnancy. Best Pract Res Clin Obstet Gynaecol 2015;29:741-753.
4 Diallo I, Lamon A, Shamsaldin A, et al: Estimation of the radiation dose delivered to any point outside the target volume per patient treated with external beam radiotherapy. Radiother Oncol 1996;38:269-271.

5 Van der Giessen PH: Measurement of the peripheral dose for the tangential breast treatment technique with Co-60 gamma radiation and high energy X-rays. Radiother Oncol 1997;42:257-264.
6 Mazonakis M, Varveris H, Damilakis J, et al.: Radiation dose to conceptus resulting from tangential breast irradiation. Int J Radiat Oncol Biol Phys 2003;55:386-391.

7 Scharwächter C, Röser A, Schwartz CA, et al.: Prenatal radiation exposure: dose calculation. Rofo 2015;187: 338-346.

8 International Commission on Radiological Protection: Biological effects after prenatal irradiation (embryo, foetus). Ann ICRP 2003;33:205-206. 
9 Loibl S, Schmidt A, Gentilini O, et al.: Breast cancer diagnosed during pregnancy: adapting recent advances in breast cancer care for pregnant patients. JAMA Oncol 2015;1:1145-1153.

10 Ivaldi GB, Leonardi MC, Orecchia R, et al.: Preliminary results of electron intraoperative therapy boos and hypofractionated external beam radiotherapy after breast-conserving surgery in premenopausal women. Int J Radiat Oncol Biol Phys 2008;72:485-493.

11 Veronesi U, Orecchia R, Maisonneuve P, et al.: Intraoperative radiotherapy versus external radiotherapy for early breast cancer (ELIOT): a randomised controlled equivalence trial. Lancet Oncol 2013;14:12691277.

12 Galimberti V, Ciocca M, Leonardi MC, et al.: Is electron beam intraoperative radiotherapy (ELIOT) safe in pregnant women with early breast cancer? In vivo dosimetry to assess fetal dose. Ann Surg Oncol 2009; 16:100-105.

13 Azim H Jr, Gentilini O, Locatelli M, et al.: Managing pregnant women with cancer: personal considerations and a review of the literature. Ecancermedicalscience 2011;5:204.

14 Bednarz B, Xu XG: A feasibility study to calculate unshielded fetal doses to pregnant patients in 6-MV photon treatments using Monte Carlo methods and anatomically realistic phantoms. Med Phys 2008;35: 3054-3061.

15 Antolak JA, Strom EA: Fetal dose estimates for electron-beam treatment to the chest wall of a pregnant patient. Med Phys 1998;25:2388-2391.

16 Gentilini O, Cremonesi M, Trifiro G, et al.: Safety of sentinel node biopsy in pregnant patients with breast cancer. Ann Oncol 2004;15:1348-1351.

17 Sechopoulos I, Suryanarayanan S, Vedantham S, et al. Radiation dose to organs and tissues from mammography: Monte Carlo and phantom study Radiology 2008; 246:434-443.
18 Ngu SL, Duval P, Collins C: Foetal radiation dose in radiotherapy for breast cancer. Australas Radiol 1992; 36:321-322.

19 Inouye M, Darmanto W, Tamaru M, et al.: Fractionated-dose effect of $\mathrm{X}$-irradiation on the induction of neural tube defects in mice. Environ Med 1997;41:4042.

20 Kal HB, Struikmans H: Radiotherapy during pregnancy: fact and fiction. Lancet Oncol 2005;6:328-333.

21 Amant F, Deckers S, Van Calsteren K, et al.: Breast cancer in pregnancy: recommendations of an international consensus meeting. Eur J Cancer 2010;46:31583168.

22 Nakagawa K, Aoki Y, Kusama T, et al.: Radiotherapy during pregnancy: effects on fetuses and neonates. Clin Ther 1997;19:770-777.

23 Glasgow G: Gestational carcinoma of the female breast. Curr Prob Cancer 1983;7:25-29.

24 Peccatori FA, Azim HA Jr, Orecchia R, et al.: Cancer, pregnancy and fertility: ESMO Clinical Practice Guidelines for diagnosis, treatment and follow-up. ESMO Guidelines Working Group. Ann Oncol 2013;24(suppl 6):vi160-70.

25 Sutlief AG: Protection and measurement in radiation therapy. Health Phys 2015;108:224-241.

26 International Commission on Radiological Protection: Pregnancy and medical radiation. Ann ICRP 2000; 30:iii-viii, 1-43.

27 Osei EK, Darko J: Foetal radiation dose and risk from diagnostic radiology procedures: a multinational study. ISRN Radiol 2012;25:1-7.

28 Health Protection Agency (HPA): Protection of pregnant patients during diagnostic medical exposures to ionising radiation: advice from the health protection agency. Documents of the Health Protection Agency: Radiation, Chemical and Environmental Hazards RCE 9, The Royal College of Radiologists and the College of Radiographers, 2009.
29 Stovall M, Blackwell CR, Cundiff J, et al.: Fetal dose from radiotherapy with photon beams: report of AAPM Radiation Therapy Committee Task Group No. 36 (review). Med Phys 1995;22:63-82.

30 Maarouf M, Treuer H, Kocher M, et al.: Radiation exposure of extracranial organs at risk during stereotactic linac radiosurgery. Strahlenther Onkol. 2005;181:463467.

31 Leonardi MC, Maisonneuve P, Mastropasqua MG, et al.: How do the ASTRO consensus statement guidelines for the application of accelerated partial breast irradiation fit intraoperative radiotherapy? A retrospective analysis of patients treated at the European Institute of Oncology. Int J Radiat Oncol Biol Phys 2012;83 806-813.

32 Leonardi MC, Maisonneuve P, Mastropasqua MG, et al.: Accelerated partial breast irradiation with intraoperative electrons: using GEC-ESTRO recommendations as guidance for patient selection. Radiother Oncol 2013;106:21-27.

33 Chen Z, King W, Pearcey R, et al.: The relationship between waiting time for radiotherapy and clinical outcomes: a systematic review of the literature. Radiother Oncol 2008;87:3-16.

34 Peccatori FA, Azim HA Jr, Scarfone G, et al.: Weekly epirubicin in the treatment of gestational breast cancer (GBC). Breast Cancer Res Treat 2009;115:591-594.

35 Cardonick E, Bhat A, Gilmandyar D, et al.: Materna and fetal outcomes of taxane chemotherapy in breast and ovarian cancer during pregnancy: case series and review of the literature. Ann Oncol 2012;23:30163023 